\title{
Implantación de sistemas integrados para una gestión eficiente de los recursos en el ámbito hospitalario
}

\author{
Implementation of integrated systems for efficient resource \\ planning in hospitals
}

\author{
Dr. Bernabé Escobar Pérez, ${ }^{\mathrm{I}}$ Dr. Tomás Escobar Rodríguez, ${ }^{\mathrm{II}}$ Dr. Pedro \\ Monge Lozano ${ }^{\text {II }}$ \\ I Universidad de Sevilla. Sevilla, España. \\ II Universidad de Huelva. Huelva, España.
}

\section{RESUMEN}

Objetivo: evaluar el impacto de la implantación de Sistemas de Información Integrados para la planificación de recursos empresariales en hospitales, mediante el análisis de su diseño y utilización, con especial atención a la satisfacción de los usuarios.

Métodos: investigación cualitativa basada en un estudio de caso longitudinal. Para la recogida de la información se utilizaron las técnicas de observación no participante, análisis de documentación interna, entrevistas individuales y en grupo y conversaciones informales. La unidad de análisis fue una Fundación Hospitalaria de la Comunidad Autónoma de Madrid seleccionada de manera intencional por su adecuación al objetivo del trabajo y por la facilidad de acceso a la información.

Resultados: las expectativas previas de los responsables del hospital se cumplieron razonablemente. Se consiguió un sistema único con distintos módulos en el que la información está integrada. Los usuarios están satisfechos con el funcionamiento del sistema, especialmente el personal clínico. Gracias a la implicación de la dirección y de los mandos intermedios, se minimizaron las dificultades derivadas de los roces entre la cultura clínica y la administrativa durante el proceso de implantación y en la utilización posterior del sistema. Conclusiones: la aplicación de Sistemas de Información Integrados en los hospitales no solo es posible, sino que resulta muy conveniente. Para la Fundación Hospitalaria supone una mejora de la eficacia en la gestión clínico asistencial y un aumento de la eficiencia en la gestión económico financiera. La implantación generalizada de estos sistemas permitiría establecer comparaciones entre distintos hospitales y avanzar hacia la gestión integral del sistema sanitario.

Palabras clave: Sistemas de Información Integrados (Enterprise Resource Planning), gestión hospitalaria. 


\section{ABSTRACT}

Objective: to evaluate the impact of the setting up of the enterprise resource planning in hospitals, by analysing their design and implementation, paying special attention to the user satisfaction.

Methods: qualitative research study based on a longitudinal case study in which the techniques of internal documentation analysis, non-participant observation, individual and group interviews and informal talks were used to collect data. The analysis unit was selected because of its suitability for the objective of the paper and easy access to the necessary information. Specifically, the study has been conducted in «Fundación Hospitalaria de la Comunidad de Madrid (FHM)».

Results: the hospital management's prior expectations of the system were fulfilled to a reasonable degree. A single system, with different modules in which the information is integrated, has been achieved. The users, especially the clinical staff, are satisfied with the operation of the system. Thanks to the involvement of the senior and middle managers, it has been possible to minimise the difficulties arising from confrontation between the clinical and the administrative cultures during the process of the setting-up and subsequent utilisation of the system.

Conclusions: the implementation of enterprise resource planning in hospitals is not only possible but also very advantageous. For the Fundación Hospitalaria de Madrid, it represents not only improved effectiveness in clinical assistance management but also higher efficiency in economic and financial resources management. If these systems were established generally, it would be possible to make constructive comparisons between different hospitals, and to progress towards the integral management of the health-care system.

Key words: Enterprise Resource Planning (ERP), hospital management.

\section{INTRODUCCIÓN}

Los profundos cambios acaecidos en las últimas décadas han hecho aumentar el interés por el funcionamiento y el control del sector público que cuenta con recursos limitados con los que satisfacer las cada vez mayores necesidades sociales. Esto es especialmente importante en el sector sanitario, dadas sus características particulares. ${ }^{1}$ Esta circunstancia exige una gestión responsable y una eficaz respuesta a las demandas planteadas. ${ }^{2}$ Por otra parte, la crisis económica actual ha agravado aún más esta situación, haciendo aún más necesario el control del gasto público sanitario.

Por ello, los hospitales públicos en España están cada vez más centrados en la gestión, ${ }^{3}$ siendo las prácticas de control de gestión que utilizan muy similares, ${ }^{4,5}$ ya que para conseguir controlar el gasto, entre otras cuestiones, los responsables del sistema sanitario requieren herramientas útiles que posibiliten una gestión eficiente de los recursos. ${ }^{6}$

Considerando sus características específicas, ${ }^{7}$ entre estas herramientas podrían encontrarse los sistemas para la planificación de recursos empresariales (Enterprise Resource Planning, por sus siglas en inglés; en lo adelante, ERP). De hecho, existen numerosas evidencias de implantación de sistemas ERP que han permitido conseguir mejoras considerables para las empresas. ${ }^{8}$ 
En este sentido, es conveniente conocer los efectos derivados de la implantación y uso de los ERP en el sector público, ${ }^{9}$ con especial atención a cómo y por qué determinadas variables influyen en este proceso. Así, una mayor comprensión de estos ayudaría a superar los obstáculos que encuentran estas innovaciones en la práctica y facilitaría su divulgación y la implantación de proyectos similares.

Por ello, este trabajo plantea como objetivo principal, evaluar el impacto de la introducción de sistemas ERP en hospitales, a través del análisis de su diseño, implantación y utilización con especial atención a la satisfacción de los usuarios.

\section{MÉTODOS}

\section{Diseño del estudio}

Para realizar el estudio se ha utilizado una técnica de investigación cualitativa y explicativa, basada en un estudio de caso y longitudinal. ${ }^{10}$ El motivo de emplear el estudio de caso como estrategia de investigación se debe a que el diseño, implantación y utilización de un ERP constituye un fenómeno complejo que no puede ser plenamente entendido aisladamente, requiriéndose un análisis dentro de la realidad empresarial. ${ }^{11} \mathrm{~A}$ diferencia de otros métodos de investigación empírica, el estudio de caso permite analizar un fenómeno contemporáneo en su contexto real, cuando las fronteras entre ambos no están claramente diferenciadas y cuando se utilizan múltiples fuentes de evidencia. ${ }^{12}$ El estudio de caso permite observar la mayoría de las variables relevantes de una realidad concreta, al tiempo que considera el contexto como parte esencial del fenómeno bajo análisis.

\section{Selección del caso}

Atendiendo al objetivo planteado, la selección de la unidad de análisis en nuestro estudio se ha realizado de manera intencionada. ${ }^{13} \mathrm{El}$ estudio de caso se hizo en una Fundación Hospitalaria (en adelante, FHM), situado en la Comunidad Autónoma de Madrid, por ser un hospital público que posee autonomía tanto administrativa como económica y de gestión, lo que obliga a la Dirección a buscar tanto la eficacia, como la eficiencia en la gestión. Además, la apertura del hospital coincidió en el tiempo con la implantación del ERP por lo que no ha existido una resistencia previa al cambio. También ha sido determinante la buena disposición y la cooperación que todos los estamentos de la institución prestaron para el desarrollo del estudio.

\section{Técnicas de recogida de información}

Las técnicas con las que se obtuvieron las evidencias necesarias para realizar el estudio fueron:

Observación directa no participante. Para apreciar el funcionamiento del hospital en general y de cada uno de los servicios en particular, para de esta forma conocer las instalaciones, el personal, la casuística, la gestión, entre otros aspectos.

Análisis de documentación interna. Para subrayar la oferta presentada por la empresa encargada de la implantación del ERP, el informe de evaluación de las ofertas presentadas al concurso, la memoria de actividades, los estatutos y el plan de empresa de la FHM.

http://scielo.sld.cu 
Entrevistas. Tanto individuales como en grupo, con responsables de las áreas Médica, Enfermería y Económico-Financiera.

Conversaciones informales. Ayudaron a crear un ambiente distendido evitando comportamientos protocolarios o formales que pudieran afectar la obtención de la información que se deseaba recabar.

\section{Análisis de los datos y calidad de la información}

Analizado el contenido de la información procedente de las entrevistas individuales y de los documentos, se identificaron y codificaron un total de 21 categorías de primer orden. Estas, con forma de preguntas, dirigen la atención hacia alguna cuestión que debe ser analizada dentro del ámbito del estudio, ya que cada una de las categorías comprende un determinado tipo de evidencia. Tras identificar y codificar las distintas categorías de primer orden, se realizó una categorización temática de segundo orden, como paso previo para obtener las conclusiones, resultando las siguientes:

Proceso de Implantación. Engloba las ventajas del ERP en cuanto a centralización de toda la información en una sola base de datos y que su introducción se realiza de una sola vez, motivos o factores que impulsaron a los miembros de la FHM para optar, desde un principio, por un ERP para el hospital, así como el papel de los directivos en su diseño.

Gestión de la Implantación. Comprende la implicación por parte del personal (Dirección General, resto de direcciones y personal), si en algún momento hubo inadaptación del personal hacia la nueva forma de trabajo y el proceso de formación para facilitar a todos los trabajadores el manejo de la aplicación.

Funcionalidad del Sistema. En ella se engloba la información sobre las facilidades o dificultades que surgen al utilizar el ERP y además si su rendimiento satisface a todas las áreas funcionales.

Evolución del Sistema. Permite explicar por qué la FHM optó por realizar una migración modular, así como por qué se ha llevado a cabo el cambio, desde que se implantó el sistema ERP hasta la actualidad, de alguna de las aplicaciones inicialmente instaladas.

\section{RESULTADOS}

La FHM inició su funcionamiento considerando que los sistemas de información constituían el factor clave en este proceso de desarrollo estratégico, de forma que la viabilidad de la puesta en marcha del modelo de hospital, sobre todo en sus relaciones internas, tanto a nivel estratégico como funcional, estaba comprometida con el desarrollo e implantación de los sistemas de información. Como objetivo irrenunciable se estableció la integración de la información clínica y económicofinanciera.

La Dirección elaboró un pliego de prescripciones técnicas para efectuar la contratación de un ERP sobre la base de lo especificado en el Plan de Empresa. La premisa fundamental era la necesidad de dotar al hospital con un sistema integrado que permitiera la ejecución de sus funciones asistenciales y que constituyera la principal herramienta para su gestión económico-financiera. Cuando se resolvió el concurso, en ningún momento se perdió de vista que lo prioritario era implantar un

http://scielo.sld.cu 
sistema integrado, aunque desde un punto de vista operativo fue necesario adoptar distintos módulos conectados entre sí a través de interfaces.

Entre otras cuestiones, el contrato incluyó una detallada descripción del producto final requerido que consistía en un ERP con 3 módulos y 11 aplicaciones. Cada uno de los módulos posee, en su versión estándar, un conjunto de interfaces para el intercambio interno de información. En cambio, no sucedió lo mismo con las distintas aplicaciones adquiridas, por lo que se hizo necesario incorporar hasta 11 interfaces (Fig.).

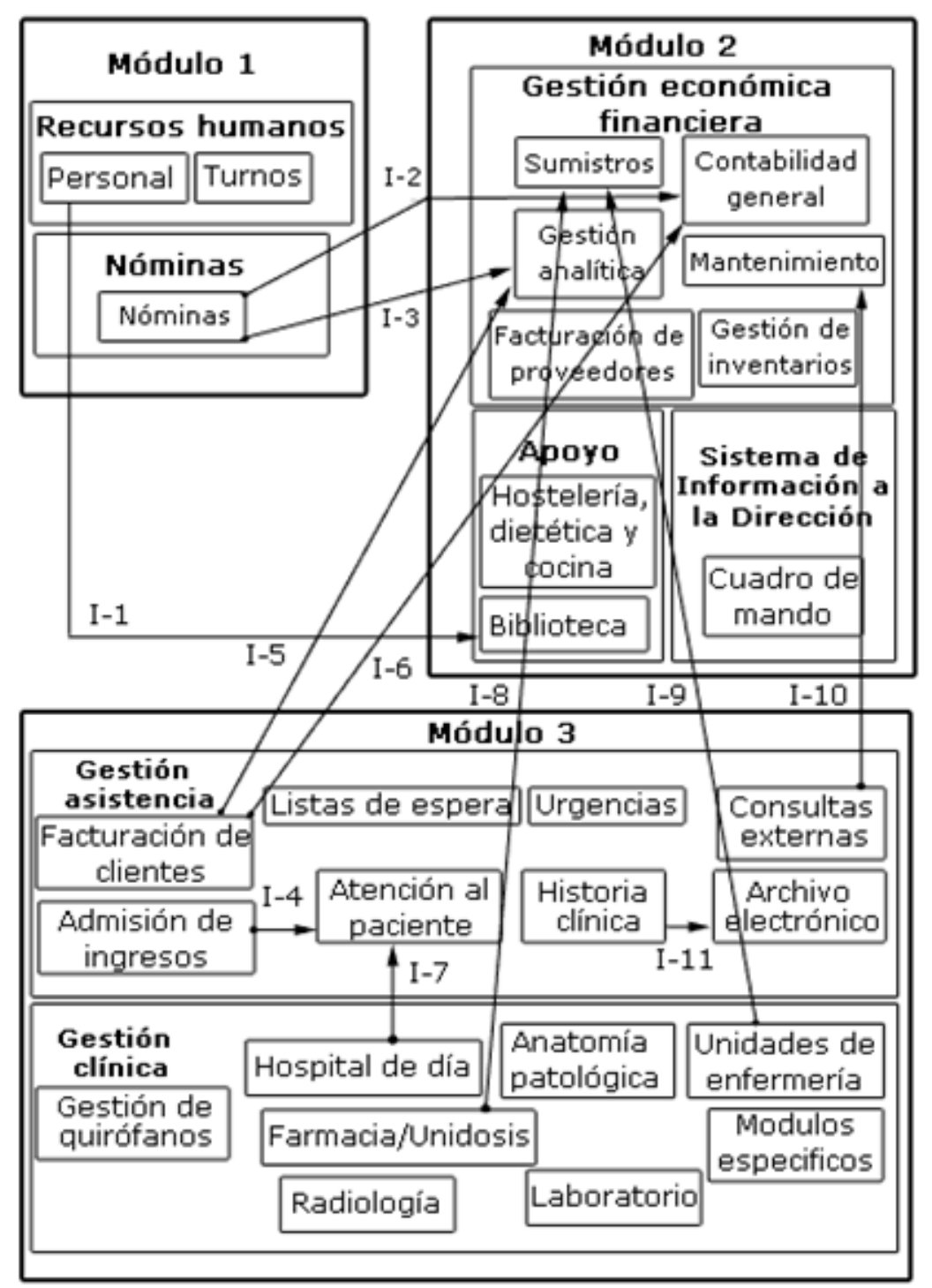

Fuente: Elaboración de los autores.

Fig. Esquema de la implantación de los módulos del sistema.

Respecto al diseño y posterior proceso de implantación, en términos generales, se cumplieron los objetivos y plazos previstos. A ello, contribuyó que la implantación del sistema fue realizada por una sola empresa, la cual suministró el hardware y el software (outsourcing). Una vez dada por concluida la implantación se produjo la puesta en marcha del sistema a la vez que comenzaba la actividad normal del hospital. 
El proceso de implantación realizado en el hospital resultó más complicado del que habitualmente se hace en otras entidades, debido a la complejidad del proceso productivo y a la escasa definición del producto final. Se realizó una implantación por etapas, lo que provocó algunos problemas en su gestión de trabajo, sobre todo en el área clínico-asistencial, pero que fueron eliminadas gracias a la colaboración del personal. Aunque en la práctica se tuvieron en cuenta las particularidades de cada una de las áreas funcionales, se antepuso la visión de la organización en su conjunto, lo que permitió una mayor integración de la información.

De las entrevistas se derivó que el principal factor que influyó en los miembros de la FHM para implantar un ERP fue su experiencia previa que les permitió comprobar que cuando no se compartía información entre las distintas áreas funcionales del hospital, se producían islas informativas que impedían realizar una buena gestión.

Entre los objetivos establecidos se encontraban mejorar la gestión mediante el uso de la informática como herramienta, disponer de una información fiable y accesible en todo momento manteniendo el principio de unicidad de datos e infundir entre el personal la idea de gestión de la información, entre otros. Asimismo, los miembros de la FHM pretendían confeccionar un protocolo de tratamientos y un perfil de costos estándar, mediante el desarrollo de un sistema de protocolos clínicos (clinical partways). Por tanto, las expectativas iniciales de los responsables eran elevadas y, sobre todo en el área asistencial constituyeron un auténtico reto. Quizá por ello, actualmente los entrevistados se muestran bastante satisfechos con el ERP que les permitió la unicidad de datos en casi todas sus actividades.

Según la evidencia obtenida, la implicación de la alta dirección fue muy importante desde la misma creación de la FHM, ya que fue planteado como un objetivo prioritario. Esto proporcionó un importante impulso al proyecto. Con posterioridad, una vez que el hospital comenzó a funcionar, la alta dirección tuvo que hacer frente a las prioridades propias de la actividad cotidiana del hospital, por lo que necesariamente se redujo la atención que prestaba al ERP. Ante esta circunstancia, los mandos intermedios tomaron la iniciativa y se implicaron en mayor medida tanto en tareas formativas, como en la resolución de conflictos. De hecho, cuando existieron conflictos de intereses, han sido ellos los encargados de mediar entre las partes. Algunas de estas situaciones pudieron ser debidas a la tradición y diferencias culturales de los distintos grupos (clínicos y gestores). Además, los cursos de formación que se impartieron al personal sanitario tuvieron como handicap la llegada masiva de dicho personal que debía incorporarse de inmediato a sus puestos de trabajo. Por lo tanto, la duración de los mismos fue insuficiente y se hizo necesaria la participación de los mandos intermedios, que sí habían recibido la formación necesaria, para ser ellos los que realmente formaran al personal sanitario sobre el uso del sistema.

No menos importante ha sido el compromiso y la implicación del resto del personal, porque en última instancia, son los encargados de introducir los datos para que el ERP pueda suministrar una información fiable. En función de ello, podemos concluir que a pesar de la existencia de grupos con distinta cultura organizativa, se ha conseguido una integración más que razonable de la información.

\section{DISCUSIÓN}

Este trabajo evalúa el impacto de los sistemas ERP en el ámbito hospitalario, a través del análisis de su diseño, implantación y utilización, con especial atención a la satisfacción de los usuarios.

http://scielo.sld.cu 
El análisis conjunto de los resultados obtenidos en el estudio de caso desarrollado, permite llegar a la conclusión de que en términos generales, la implantación de los sistemas ERP en los hospitales resulta factible, tanto técnica como organizativamente.

En cuanto a sus efectos, hemos de destacar que la implantación del sistema ERP ha supuesto para la FHM tanto una mejora de su eficacia en la gestión clínicaasistencial, como un aumento de su eficiencia en la gestión económico-financiera.

Asimismo, podemos señalar que existe una satisfacción generalizada entre el personal del hospital como consecuencia del uso del ERP. Por una parte, entre el personal clínico-asistencial porque puede disponer en poco tiempo de toda la información de las pruebas realizadas a los pacientes, así como de su historial completo. Por otra, entre el personal administrativo porque supone un gran ahorro de tiempo en lo que se refiere a su operativa básica, sobre todo, en las tareas más rutinarias.

También es preciso subrayar que el sistema ERP se ha manifestado como un proyecto en permanente evolución, ya que requiere de actualizaciones y mejoras constantes para responder a las cada vez mayores demandas informativas de las distintas áreas hospitalarias. Lógicamente, esto implica la necesidad de llevar a cabo inversiones de forma constante. De hecho, ya se ha optado por realizar una migración que permita mejorar el rendimiento del sistema e incorpore nuevas herramientas para facilitar su uso.

Por último, nos gustaría concluir poniendo de manifiesto que la implantación generalizada de sistemas ERP en los distintos sistemas sanitarios públicos de nuestro país es posible y muy conveniente y no solo posibilitaría establecer comparaciones relevantes entre distintos hospitales, sino que también permitiría avanzar hacia una gestión integral del sistema sanitario público.

\section{REFERENCIAS BIBLIOGRÁFICAS}

1. Lluch E. Costos crecientes y éxitos sanitarios públicos. Revista de Administración Sanitaria. 1997;1(1):35-45.

2. Aparisi JA. La eficacia del cuadro de mando integral en el control estratégico de las entidades públicas. Valencia: Ed. Sindicatura de Comptes de la Comunitat Valenciana; 2008.

3. Villalbía J, Guixa J, Casasa C, Borrella C, Durana J, Artazcoza L, et al. El Cuadro de Mando Integral como instrumento de dirección en una organización de salud pública. Gacet Sanit. 200;21(1):60-5.

4. Carretero L. Herramientas para la gestión clínica. Rev Gestión Hospitalaria. 1999;11:15-21.

5. Asenjo MA. Las claves de la gestión hospitalaria. Barcelona: Ed. Gestión 2000, S.A.; 1999.

6. Rubin P. The right stuff...meeting your customer needs. Hosp Materiel Manag Quart. 1999;21(2):12-7.

http://scielo.sld.cu 
7. Akkermans $\mathrm{H}$, Van Helden K. Vicious and virtuous cycles in ERP implementation: a case study of interrelations between critical success factors. Eur J Informat Systems. 2002;11:35-46.

8. Escobar B, Cullen J, González JM. Impacts of the Implementation of ERP Systems on Cash Management: The Redesign of Treasury Processes. Internat J Digital Account Res. 2004;4:31-55.

9. Cavalluzzo KS, Ittner CD. Implementing performance measurement innovation: evidence from government. Accounting Organizations Soc. 2004;29:243-67.

10. Yin RK. Case study research: Design and methods. California, Beverly Hills: Ed. Sage Publications; 1984.

11. Al-Mashari M, Al- Mudimigh A. ERP implementation: lessons from a case study. Information Technology \& People. 2003;16(1):21-34.

12. Yin RK. Applications of case study research. London, New Cambridge, Beverly Hills: Ed. Thousand Oaks, Sage Publications Cop.; 1993.

13. Einsenhardt K. Building theories from case study research. Academy Management Rev. 1989;14:532-50.

Recibido: 20 de abril de 2011.

Aprobado: 23 de noviembre de 2011.

Tomás Escobar Rodríguez. Facultad de Ciencias Empresariales. Universidad de Huelva. Plaza de la Merced. 21002 Huelva, España.

Correo electrónico: tescobar@uhu.es bescobar@us.es, monge@uhu.es

http://scielo.sld.cu 